\title{
COMMENTARY
}

\section{The Impact of Implicit Biases in Pharmacy Education}

\author{
Lalita Prasad-Reddy, PharmD, MS, ${ }^{a}$ Paul Fina, PharmD, ${ }^{a}$ Daniel Kerner, PharmD, ${ }^{\text {a }}$ Bianca Daisy-Bell, \\ PharmD ${ }^{\mathrm{b}}$ \\ ${ }^{\text {a }}$ Chicago State University College of Pharmacy, Chicago, Illinois \\ ${ }^{\mathrm{b}}$ Logan and Seiler Incorporated, Sikeston, Montana
}

Corresponding Author: Lalita Prasad-Reddy, Chicago State University, College of Pharmacy, 9501 South King Drive, Chicago, IL 60628. Tel: 402-250-0680. Email: 1prasad@csu.edu

Submitted December 17, 2020; accepted February 23, 2021

Keywords: Bias, implicit, unconscious, inequity

\section{BACKGROUND}

In the United States, the very infrastructure of our healthcare system gives rise to healthcare disparities. ${ }^{1}$ Lower access to healthcare institutions, pharmacies, and qualified practitioners is prevalent among African Americans, Hispanics, and Native Americans. ${ }^{1}$ However, other elements such as implicit bias contribute to the widening gap in inequitable healthcare delivery. Implicit bias is defined as the attitudes or stereotypes that affect our understanding, actions, and decisions in an unconscious manner. ${ }^{2}$ Implicit attitudes and behaviors are not readily identified and are formed through previous experiences. In 2012, the Institute of Medicine concluded that "some evidence suggests that bias, prejudice, and stereotyping on the part of healthcare providers may contribute to differences in care." ${ }^{3}$ While it remains unclear if bias has a direct correlation to patient outcomes, all healthcare professionals should be committed to an ethos of altruistic service. Therefore, knowledge on implicit biases and how they may affect one's practice is essential in providing non-discriminatory care.

Healthcare disparities exist worldwide among socioeconomic classes, ethnic populations, sexual orientations, and educational backgrounds among other characteristics. Evidence demonstrates that implicit biases play a role in patient marginalization. ${ }^{4}$ Studies have evaluated the presence of implicit biases in healthcare providers, and the majority suggest that most healthcare providers exhibit some degree of implicit bias towards patients. ${ }^{4}$ Determining whether those biases have a direct correlation to patient care outcomes is more challenging. Although the literature has demonstrated the presence of implicit biases in healthcare professionals, there is a lack of information on the presence of implicit biases in healthcare education.

\section{Assessing Implicit Bias}

As implicit bias is an unconscious belief that impacts our behaviors, individuals are usually unaware of their own biases. Due to this lack of awareness, it is incredibly difficult for an individual to identify their implicit biases using selfreflection alone. Various strategies have been developed that can aid an individual in assessing implicit biases. ${ }^{5}$ The Implicit Association Test (IAT) is commonly used and considered by many to be the most effective tool for measuring implicit bias. ${ }^{6}$ Prior to beginning the test, the examinee is shown the list of words and/or pictures that will be used in the test as well as their associated category. The words and/or pictures are divided into different categories: two contrasting concepts (eg, young, old) and two contrasting evaluations or stereotypes (eg, athletic, clumsy). The examinee is instructed to match words or pictures from a concept group with words or pictures from an evaluation group, or vice versa. Examinees are instructed to match different pairs of groups in different parts of the test. The principle behind the IAT is that responses will occur more quickly if the examinee has an underlying belief that evaluation words are more associated with one concept over the other. For example, if an examinee has an implicit bias to think young people are more athletic, they will pair words from those categories quicker than words from the young and clumsy categories. Based on the time taken to make selections, the test measures the strength of association. Upon completion of the test, the examinee is provided with their results and an explanation of any identified implicit biases. The results also label scores as "slight", "moderate", or "strong" to indicate the strength of implicit preference. These results provide insight into one's implicit biases. Other tests and neurological imaging (ie. functional MRI) have been used to supplement the IAT to assess implicit biases. ${ }^{5,6}$ 
While these objective strategies may divulge information regarding bias, they do not examine behaviors. The examination of behavior is a powerful method to combat bias by reflecting on past actions and factors leading to those actions. By examining several actions as a collective group, patterns may begin to develop which may identify implicit biases. For example, choosing to hire one individual over another does not provide enough information to conclude that implicit bias exists. Extrinsic factors, such as more experience or a history of exemplary work in a previous position, may make one candidate superior. Examining a larger sample size of decisions may uncover a pattern of implicit bias. This strategy of reviewing past behaviors has been used in previous research to identify implicit bias and its impact, and many advocates support behavior examination and reflection in addressing implicit bias. ${ }^{4,7}$

\section{Implicit Bias in Healthcare Curricula}

Unfortunately, healthcare education may contribute and cultivate the presence of implicit biases. While it is the imperative to cultivate the development of emotionally intelligent practitioners, formal education and training on the identification and mitigation of implicit biases is lacking. ${ }^{8}$

Evidence suggests that pharmacy students exhibit implicit biases. A 2018 study examined implicit biases in firstyear pharmacy students. ${ }^{9}$ Students completed the IAT and then a self-reflection analyzing whether they agreed or disagreed with the results. The majority of students, who classified themselves as White Non-Hispanic, displayed some level of preference towards Caucasians. Students who explicitly agreed with their survey results cited that biases were likely fostered by environmental influences, family, and friends. Individuals who disagreed with the results felt that the results were invalid or would not negatively affect clinical decision-making. The study demonstrated that many students are not only unaware of their biases, but at times, surprised by their unconscious beliefs. Providing these opportunities to identify and reflect on biases has been shown to build self-awareness and sensitivity.

Another study of health professional trainees included medical, pharmacy, and nursing students. ${ }^{10}$ The study evaluated students' understanding of cultural competency and investigated the presence of implicit biases. Students who identified themselves as Hispanics or non-Hispanic Blacks had improved cultural competency scores when compared to students identified as non-Hispanic Whites. Students who recognized themselves as multiracial also exhibited enhanced cultural competency. Cultural biases were recognized by the IAT, which demonstrated that overall, the students exhibited implicit race and skin tone preferences, with a stronger preference for individuals who were light skinned over dark skinned and a preference for white over black individuals.

Another study administered a questionnaire to third-year pharmacy students in a management course. ${ }^{11}$ Although there was no statistically significant differences between males and females in the number of prior business courses $(p=.16)$ or pre-test scores $(\mathrm{p}=0.13)$, females self-assessed their management scores lower than males $(\mathrm{beta}=-2.12, p$ $=.01$ ), indicating a possible gender bias in self-perceived management skills. Identification and mitigation of implicit biases within professional school curricula should be a priority for educational institutions as a method to reduce potential inequalities in care. ${ }^{2}$

\section{Implicit Bias in Higher Education Infrastructure}

Implicit bias in pharmacy academic infrastructure has been examined via multiple methods, which collectively may demonstrate bias. The most readily available statistic to review this is the diversity of faculty and students. A study by Lebovitz and colleagues examined the landscape of underrepresented minority faculty members at pharmacy schools and found that there has been an increase in Asian faculty members over 10 years (2008 to 2017) but little to no change in Black and Hispanic faculty members. ${ }^{12}$ The study further found that there was no change in the low enrollment of Black (5\% to $4 \%$ of the total) and Hispanic (2\% to $3 \%$ of the total) PhD students at pharmacy schools. Chisholm-Burns examined faculty representation and determined that from 1989 to 2009, the number of women faculty members increased from $20.7 \%$ to $45.5 \% .{ }^{13}$ However African American, Hispanic, and Native American faculty member representation remained low at approximately $11 \%, 8 \%$, and $6 \%$ for assistant, associate, and full professors respectively. Collectively, this means that faculty representation of underrepresented minorities has been low since 1989.

Pharmacy institutions have been proactive in trying to mitigate implicit bias during the admissions process. McLaughlin and colleagues recommend pharmacy personnel reviewing applications undergo implicit bias training. ${ }^{14}$ The University of Minnesota administered the Intercultural Development Inventory to all College of Pharmacy personnel, with those involved in admissions receiving additional training. ${ }^{15}$ They found an immediate impact of increased awareness, knowledge, and skills with the review of applicants. ${ }^{15}$ Colleges of medicine have also endorsed such training methods, and have found that training does result in a more diverse, incoming student body. ${ }^{16}$

Unconscious biases also exist within training materials. While we attempt to educate students on the importance of cultural competence and caring for diverse patients, educational materials largely under-represent minority populations. In one analysis of textbooks utilized in medical schools, there was a large overrepresentation of light skin tone images 
when compared to dark skin tone images which did not reflect the population distribution. ${ }^{17}$ Lastly, Vyas et al. warn of the dangers of race-baseed medicine which is commonplace within clinical practice. ${ }^{18}$ Algorithms which guide treatment selection based upon racial characteristics, ingrain bias into the system, such as when determining treatment for hypertension based upon race alone. The pervasive use of these algorithms may potentiate the differences in healthcare outcomes leading to inequities in care.

\section{Tools to Address Implicit Bias}

\section{Educational Interventions}

Educators can utilize a variety of mediums to address implicit biases. While evidence is limited, didactic teaching, incorporating the IAT, reflection, and experiential learning have been utilized within medical education. ${ }^{19}$ Studies have also demonstrated that students believe implicit bias education should occur in pre-clinical, didactic years, prior to exposure to patient care arenas. ${ }^{20}$ Didactic instruction about bias may be the initial step for a learner in recognizing biases. Colleges can begin by supplementing cultural competency curriculums with implicit bias focuses. While cultural competency has many similarities, educators must recognize and instruct about fundamental cross-cultural issues as opposed to cultural group tendencies. Cultivating a safe, non-punitive learning environment is also essential for optimal learning due to the sensitive nature of the topic. ${ }^{19,21}$ Moderators should be trained to skillfully provide non-judgmental sessions, as optimal quality is achieved when feedback is given by an experienced moderator. ${ }^{21,22}$

Experiential learning plays a significant role in reducing bias. ${ }^{23}$ Students should be exposed to diverse groups of learners and patients as a tool to mitigate bias. Delivery of care to diverse groups has been shown to have a significant positive impact on reducing bias. ${ }^{24}$ To avoid external variability among experiences, simulations have also been utilized to address unconscious bias. ${ }^{25}$

Self awareness and reflection of biases is key to development in this area. By utilizing previously mentioned tools, learners can reflect on their own subconscious beliefs to address the issue, including emphasizing to subjects that that beliefs are malleable and can be changed. ${ }^{26}$ Examples of interventions that have been shown to reduce bias can be found in table $1.21,23,25,27,28$

\section{$\underline{\text { Reflection }}$}

An aptitude for lifelong learning is essential in healthcare. As students progress into the healthcare field, feedback volume tends to be sporadic, therefore practitioners must learn the value of self-reflecting. ${ }^{28} \mathrm{~A}$ variety of reflecting activities have been utilized to bring awareness about unconscious bias. ${ }^{21,27}$ The value of reflection must be instilled early within the healthcare curriculum for maximum effectiveness. ${ }^{21}$ Research has also demonstrated that encouraging reflection after IAT assessment may enhance subject understanding. When given in conjunction with the IAT, students reported newfound insight into unperceived biases, providing them the tools to be more perceptive in the future. ${ }^{9,20}$

Prior to initiating reflection, educators must explain the "why" behind reflecting, so students understand the motivation for self-reflection. Educators must be skillfully trained, must cultivate a safe space for students to face these unconscious perceptions and feedback delivery should be considered when assessing. ${ }^{22,28}$ Optimal quality and outcomes is achieved when feedback is given by an experienced moderator. $\underline{\underline{11}}$

\section{Institutional Interventions}

Organizations are recognizing the threats of implicit biases in their overall commitment to diversity, sustainability, and health. Institutional interventions are aimed at reducing implicit bias within organizational settings. The main theme of these interventions is the development of standardized measurements for hiring, performance evaluations, and promotion requirements, and methods to foster diverse and inclusive environments. Institutions should support implicit bias training to standardize criteria used in these situations to allow for more objective decisions to be made and reduce the opportunity for implicit bias to influence decisions. ${ }^{2}$ Professional organizations that govern healthcare education such as the American Association of Medical Colleges (AAMC) are sponsoring initiatives that highlight the importance of including scholarly approaches within the curricula to mitigate these implicit biases, and should be endorsed by other professional educational academies. AAMC provides readily available education on implicit bias to support colleges of medicine and healthcare providers. These initiatives include how to address implicit bias during student and faculty interview recruitment, establishing successful mentoring programs for faculty to cope with their own biases, and how to decrease microaggressions and enhance cultural competency, all of which will foster success in diversity and inclusion initiatives. ${ }^{2}$ Organizations such as AACP can adopt such training programs for academic leaders and faculty to foster sustainable change and contribute to a more diverse and inclusive atmosphere for pharmacy academia. 


\section{CONCLUSION}

The presence of implicit biases is commonplace in healthcare. More research is needed to identify the optimal method to identify implicit biases and address it within the educational arena. Evidence shows that didactic and experiential education should provide learners opportunities to recognize and reflect on implicit biases in a nonjudgmental environment. Since we are all products of our personal environmental experiences, knowledge of our own implicit biases will ensure we move to providing equitable care for all.

\section{REFERENCES}

1. Jackson CS, Gracia JN. Addressing health and health-care disparities: the role of a diverse workforce and the social determinants of health. Public Health Rep. 2014;129 Suppl 2(Suppl 2):57-61.

2. Association of American Medical Colleges. Proceedings of the diversity and inclusion innovation forum: unconscious bias in academic medicine. 2017. Accessed January 1, 2021. https://store.aamc.org/downloadable/download/sample/sample_id/168/

3. Institutional and Policy-Level Strategies for Increasing the Diversity of the U.S. Healthcare Workforce. In the Nation's Compelling Interest: Ensuring Diversity in the Health-Care Workforce. Washington (DC): National Academies Press (US); 2004.

4. Hall WJ, Chapman MV, Lee KM, et al. Implicit racial/ethnic bias among health care professionals and its influence on health care outcomes: a systematic review. Am J Public Health. 2015;105(12):e60-e76.

5. Kirwan Institute for the Study of Race and Ethnicity. Implicit Bias Module Series. The Ohio State University. Published 2018. Accessed August 12, 2020. http://kirwaninstitute.osu.edu/implicit-bias-training/.

6. Project Implicit. Project Implicit Website. Available Published 2011. Accessed August 12, 2020. at https://implicit.harvard.edu/implicit/user/pih/pih/thescientists.html.

7. Rooth D-O. Automatic associations and discrimination in hiring: real world evidence. Labour Econ. 2010;17(3):523-534.

8. Laraque-Arena D. Meeting the challenge of true representation in US medical colleges. JAMA Netw Open. 2019;2(9):e1910474. Published 2019 Sep 4..

9. Avant ND, Weed E, Connelly C, Hincapie AL, Penm J. Qualitative analysis of student pharmacists' reflections of Harvard's race implicit association test. Curr Pharm Teach Learn. 2018;10(5):611-617.

10. White-Means S, Zhiyong Dong, Hufstader M, Brown LT. Cultural competency, race, and skin tone bias among pharmacy, nursing, and medical students: implications for addressing health disparities. Med Care Res Rev. 2009;66(4):436-455.

11. Painter JT, Castleberry AN. Gender differences in student preconceptions of a pharmacy management course. Poster presented at $115^{\text {th }}$ Annual Meeting of the American Association of Colleges of Pharmacy; 2014; Grapevine, Texas.

12. Lebovitz L, Swaan PW, Eddington ND. Trends in research and graduate affairs in schools and colleges of pharmacy, part 3: underrepresented minorities. Am J Pharm Educ. 2020;84(5):7641.

13. Chisholm-Burns MA, Spivey CA, Billheimer D, et al. Multi-institutional study of women and underrepresented minority faculty members in academic pharmacy. Am J Pharm Educ. 2012;76(1):7.

14. McLaughlin JE, Bush AA, Friedman AD, Lai SK. Immersive research experiences for high school students aimed at promoting diversity and visibility in pharmacy education. Am J Pharm Educ. 2020;84(3):ajpe7589.

15. Garza OW, Johnson LA, Buncher O, et al. Advancing diversity in the pharmacy pipeline through a respectful holistic admissions process. Poster presented at : 117th Annual Meeting of the American Association of Colleges of Pharmacy; 2016; Anaheim, California.

16. Capers Q 4th, Clinchot D, McDougle L, Greenwald AG. Implicit racial bias in medical school admissions. Acad Med. 2017;92(3):365-369..

17. Louie P, Wilkes R. Representations of race and skin tone in medical textbook imagery. Soc Sci Med. 2018;202:38-42..

18. Vyas DA, Eisenstein LG, Jones DS. Hidden in plain sight - reconsidering the use of race correction in clinical algorithms. N Engl J Med. 2020;383(9):874-882.

19. Teal CR, Gill AC, Green AR, Crandall S. Helping medical learners recognise and manage unconscious bias toward certain patient groups. Med Educ. 2012;46(1):80-88.

20. Motzkus C, Wells RJ, Wang X, et al. Pre-clinical medical student reflections on implicit bias: Implications for learning and teaching. PLoS One. 2019;14(11):e0225058. Published 2019 Nov 15.

21. Teal CR, Shada RE, Gill AC, et al. When best intentions aren't enough: helping medical students develop strategies for managing bias about patients. J Gen Intern Med. 2010;25 Suppl 2(Suppl 2):S115-S118. 
22. Sukhera J, Milne A, Teunissen PW, Lingard L, Watling C. The actual versus idealized self: exploring responses to feedback about implicit bias in health professionals. Acad Med. 2018 Apr;93(4):623-629..

23. Dasgupta N, Greenwald AG. On the malleability of automatic attitudes: combating automatic prejudice with images of admired and disliked individuals. J Pers Soc Psychol. 2001;81(5):800-814.

24. Erickson J, O'Connor SE. Service learning: does it promote or reduce prejudice? In: O'Grady CR, ed. Integrating Service Learning and Multicultural Education in Colleges and Universities. Lawrence Erlbaum Associates 2000;59-70.

25. Morell VW, Sharp PC, Crandall SJ. Creating student awareness to improve cultural competence: creating the critical incident. Med Teach. 2002;24(5):532-534.

26. Dasgupta, N. Implicit attitudes and beliefs adapt to situations: a decade of research on the malleability of implicit prejudice, stereotypes, and the self-concept. Adv Exp.Soc Psychol. 2013; 47: 233-279.

27. American Academy of Family Physicians. The Everyone Project. Implicit Bias Training Facilitator Guide. Published 2019. Accessed January 1, 2021.

https://www.aafp.org/dam/AAFP/documents/patient_care/health_equity/implicit-bias-training-facilitatorguide.pdf

28. Sandars J. The use of reflection in medical education: AMEE Guide No. 44. Med Teach. 2009;31(8):685-695. 
Table 1. Intervention Activities to Reduce Implicit Bias

Intervention Associated Activities

Practicing Mindfulness Mindfulness techniques have been shown to reduce the likelihood that implicit biases will be activated in the mind, or enhance the ability of the subject to control biases when in patient care situations.

Self-Awareness

Individuals should complete the IAT and then reflect on identified Training/Self

Reflection Training biases to provide opportunity for self awareness and reflection. When given in conjunction with the IAT, this insight can provide tools for students to be more perceptive in the future. ${ }^{4}$

Activating Goals

The individual should identify goals that promote fairness and equality and associate the goals with tasks that individuals perform when interacting with minority groups.

Stereotype replacement The individual should aim to replace stereotypical thoughts or responses with non-stereotypical ones; by collecting information that is opposite of cultural stereotypes one can encourage development of new associations.

Counter-stereotype

The individual should imagine outgroup members who have opposite imaging characteristics of a preconceived stereotype in an effort to break associations and preconceived notions.

Case Studies or The individual should analyze case studies to determine how implicit Observing implicit bias biases played a role in outcomes and reflect on how different approaches could have potentially altered the outcome.

Individuating

The individual should attempt to see others for their own individual characteristics, as opposed to their stereotypical characteristics (grouping).

Perspective taking The individual should aim to take on the perspective of a member in the stigmatized or marginalized group in an attempt to build empathy. 